\title{
THE THEORY OF INTEGRAL EQUATIONS
}

\author{
By H. BATEMAN.
}

[Received and Read December 14th, 1905.-Revised and enlarged January 19th, 1906.]

I. Sketch of the Subject.

II. The Integral Equation of the First Kind.

III. Reduction of the General Linear Differential Equation to an Integral Equation of the Second Kind.

IV. The Effect of Varying the Limits in an Integral Equation of the Second Kind.

V. A Certain Partial Integral Equation.

VI. Literature.

\section{Sketch of the Subject.}

Although equations involving definite integrals are to be found in the works of Abel and Gauss, it was not until quite recently that a general theory of the subject began to be constructed.

1. In the solution of a dynamical problem* Abel found it necessary to determine a function $\phi(t)$ such that, for a given arbitrary function $f(s)$,

$$
f(s)=\int_{0}^{s} \frac{\phi^{\prime}(t) d t}{\sqrt{ }(s-t)}
$$

This he succeeded in doing, showing that the more general equation

is satisfied by taking

$$
f(s)=\int_{0}^{s} \phi(t)(s-t)^{-n} d t \quad(0<n<1)
$$

$$
\phi(s)=\frac{\sin n \pi}{\pi} \int_{0}^{s} f^{\prime}(t)(s-t)^{n-1} d t .
$$

This equation has lately been examined by Goursat, + who gives the solution for the case in which $f(0)$ is not zero. Abel's formula was generalised by Sonine, $\downarrow$ but it was left for Volterra $\S$ to give the complete solution of

- "Solution de quelques problèmes à l'aide d'intégrales définies" : Mragazin for Naturvidenskaberne, Aargang 1, Bind 4 ., Christiania, 1823 ; Coll. Works (Sylow and Li甘), Vol. I., p. 11.

+ Acta Math., 1903.

$\ddagger$ Ibid., 1884 ; see also ibid., 1879.

Ann. di Mat., [2], xxv., 1897. The first result is also given by Le Roux, Ann. éc. norm., [2], xu., 1895, p. 293. 
the general equation

$$
f(y)-f(\alpha)=\int_{a}^{y} \phi(x) H(x, y) d x .
$$

His results are as follows.

If $f(y)$ and $f^{\prime}(y)$ remain finite and continuous for values of $y$ lying between $\alpha$ and $\alpha+A$, and $H(x, y)$ and $\frac{\partial H}{\partial y},=H_{2}(x, y)$, are always finite for $y>x>a, a+A>y>a$, and are integrable, and if the lower limit of the absolute value of $h(y)=H(y, y)$ is different from zero, there will exist one, and only one, finite and continuous function $\phi$, which satisfies the functional equation for values of $y$ between $a$ and $a+A$, and this function will be given by

$$
\phi(y)=\frac{f^{\prime}(y)}{h(y)}-\frac{1}{h(y)} \int_{a}^{y} f^{\prime}(x) \sum_{0}^{\infty} S_{i}(x, y) d x,
$$

where

$$
S_{0}(x, y)=H_{2}(x, y) / h(x)
$$

and

$$
S_{i}(x, y)=\int_{y}^{x} S_{i-j}(x, \xi) S_{j-1}(\xi, y) d \xi,
$$

the term "integrable" being understood to include the conditions for a change in the order of integration in the multiple integrals which occur.

If the function $H(x, y)$ becomes infinite for $y=x$, so that the equation may be written

$$
f(y)-f(a)=\int_{a}^{y} \phi(x) \frac{G(x, y)}{(y-x)^{\lambda}} d x \quad(\lambda<1),
$$

and if the function $G(x, y)$ satisfies the same kind of conditions as $H(x, y)$, and the functions $G_{2}(x, y)$ and $g(y)$ are defined as before, then there exists one, and only one, finite and continuous function $\phi$ which satisfies the functional equation for values of $y$ between $\alpha$ and $\alpha+A$, and this will be given by

$$
\phi(z)=\frac{\sin \lambda \pi}{\pi} \frac{1}{g(z)} \int_{a}^{z} f^{\prime}(x) \sum_{0}^{\infty} T(x, z) d x,
$$

which

$$
\begin{aligned}
& S_{0}(y, z)=\frac{\sin \lambda \pi}{\pi} \frac{1}{g(z)} \int_{y}^{z} G_{2}(y, \xi)\left(\frac{\xi-y}{z-\xi}\right)^{1-\lambda} \frac{d \xi}{z-y}, \\
& T_{0}(x, z)=(z-x)^{\lambda-1}, \\
& T_{i}(x, z)=\int_{z}^{x} S_{0}(\xi, z) T_{i-1}(x, \xi) d \xi .
\end{aligned}
$$


2. The equation $f(s)=\int_{a}^{b} \kappa(s, t) \phi(t) d t$,

which also dates back from Abel,"* has been called by Hilbert an integral equation of the first kind. The functions $f(s)$ and $\kappa(s, t)$ are supposed to be known, and the function $\phi(t)$ is to be determined. The process of passing from $\phi(t)$ to $f(t)$ may be regarded as a transformation, and the function $\kappa(s, t)$ may be called the generating functiont of the transformation; the theory has been developed from this point of view by Pincherle.t

An important case in which a solution of the equation can be found is furnished by Fourier's double integral, which states that, if

then

$$
g(s)=\int_{0}^{\infty} f(t) \cos \frac{\pi s t}{2} d t
$$

$$
f(s)=\int_{0}^{\infty} g(t) \cos \frac{\pi s t}{2} d t
$$

but the function $g(s)$ must be subject to certain restrictions, and this is usually the case for an integral equation of the first kind.

The beautiful simplicity of the above formula and of a number of others of a similar character§ led mathematicians to seek a general theory of such recurrence formulæ. An analogous reciprocal formula for the

" "Sur les fonotions génératrices et leurs déterminantes": Collected Works (Sylow and Lie), tome $\mathbf{~} \mathbf{}$, $\mathbf{x i}$.

$t$ In Germany the word Kern is used.

+ "Sur certaines opérations fonctionnelles" : Acta Math., x.

$\oint$ For example, if $g(s)=\int_{0}^{\infty} h(x) x^{1-s} d x$,

then

$$
2 \pi i h(y)=\int_{a-\infty i}^{a+\infty} g(s) y^{s} d s
$$

(Riemann, "Ueber die Anzahl der Primzahlen unter einer gegebene Grösse," 1859 : Gesammelte Werke, Weber, p. 140).

If

$$
\begin{aligned}
& g(s)=\int_{c} e^{s t} h(t) d t, \\
& h(t)=2 \pi i \int_{0}^{\infty} e^{-t s} g(s) d s
\end{aligned}
$$

(Pincherle, Mem. della Accad. della Sc. dell' Ist. di Bologna, Serie 4, t. vir.).

If

$$
\begin{gathered}
f(s)=\int_{0}^{\infty} J_{n}(s t) \phi(t) t d t, \\
\phi(s)=\int_{0}^{\infty} J_{n}(s t) f(t) t d t
\end{gathered}
$$

then

(Hankel, Math. Ann., Bd. vIr., p. 482, 1875 ; Sonine, Math. Ann., Bd. xvI., p. 17, 1880). 
Laplace transformation was found by Petzval,* and accordingly the integral equation of the first kind was studied in connection with the solution of linear differential equations by means of definite integrals. ${ }^{+}$

The connection with differential equations depends upon the possibility of constructing a relation

$$
P_{s}\{\kappa(s, t)\}=Q_{t}\{g(s, t)\},
$$

where $P_{s}$ is an operator such that

$$
P_{s} \int_{a}^{b} \kappa(s, t) \phi(t) d t=\int_{a}^{b} P_{s} \kappa(s, t) \phi(t) d t
$$

and

$$
P_{s}\{f(s)\}=0 \text {. }
$$

The function $\phi(t)$ must then be such that the integral

$$
\int_{a}^{b} Q_{t}\{g(s, t)\} \phi(t) d t
$$

can be directly evaluated to the value zero. If $Q_{t}$ has the form $\Sigma a_{r}(t) \frac{d^{n}}{d t^{n}}$, the function $\phi$ is a solution of the equation adjoint to $Q_{t}=0$; for then the quantity under the integral sign is a perfect differential. It appears to be generally true that, if a definite integral (4) exists, then there is a corresponding relation of the form (5), but the operators $P_{s}$ and $Q_{t}$ will not always contain differentiations only: indeed $P$ may contain a number of integrations, and either operator may contain the operator

$$
\Delta f(x) \equiv f(x+1)-f(x) .
$$

Another method of treating the equation

$$
f(s)=\int_{a}^{b} \kappa(s, t) \phi(t) d t
$$

was suggested by Volterra. $\ddagger$ If $a<s<b$ and $\kappa(s, t)$ is symmetrical in $s$ and $t$, the solution for an arbitrary $f$ can be found if it is possible to determine a function $\lambda(t, z)$ such that, for $a<t<z<b$, the integral

is independent of $z$.

$$
\int_{a}^{z} \lambda(t, z) \kappa(s, t) d t
$$

We shall return to the discussion of the integral equation of the first

- Integration der linearen Differentialgleichungen, pp. 472, 473.

$\dagger$ A list of references is given in Pincherle's book: Le operazioni distributive e le loro applicazioni all' analisi, Bologna, 1901.

‡ "Sopra una problema di elettrostatica," Rom. Linc. Trans., Ser. 3, Vol. vir. 
kind in $\S 2$, and shall pass on now to the equation which owes its origin to Neumann's method of solving the problem of Dirichlet.*

The form in which Neumann's problem is presented by Poincarét is as follows :-

Let $S$ be a closed plane curve without double points. We propose to find a double layer of moment $\phi$, such that its potential $W$ satisfies the following conditions :-

It is harmonic inside and outside $S$, and its values $V$ and $V^{\prime}$ at two points inside and outside $S$ and very close to it are connected by the relation

$$
V-V^{\prime}=\lambda\left(V+V^{\prime}\right)+2 \Phi,
$$

where $\Phi$ is a given function.

If we put $\lambda=-1$, we obtain Neumann's problem, but it is convenient to retain this parameter, as it gives additional clearness to the subsequent work.

If, now, $x=x(s), y=y(s)$ are the equations of the curve, the potential theory gives us the equations

$$
\begin{aligned}
& V(s)=\pi \phi(s)+\int_{s} \phi(t) \frac{\partial}{\partial t} \tan ^{-1} \frac{y(t)-y(s)}{x(t)-x(s)} d t, \\
& V^{\prime}(s)=-\pi \phi(s)+\int_{s} \phi(t) \frac{\partial}{\partial t} \tan ^{-1} \frac{y(t)-y(s)}{x(t)-x(s)} d t,
\end{aligned}
$$

$\phi(t)$ being the strength of the double layer.

Substituting these values in (6), we are led at once to an equation of the form

$$
f(s)=\phi(s)+\mu \int_{s} \kappa(s, t) \phi(t) d t,
$$

which has been called by Hilbert an integral equation of the second kind. Many other problems in mathematical physics lead to equations of a similar character; so that we are justified in devoting considerable attention to their study.

If we write the equation

$$
f(s)=\phi(s)-\lambda \int_{a}^{b} \kappa(s, t) \phi(t) d t
$$

in the symbolical form $f(s)=\left(1-\lambda S_{\mathrm{k}}\right) \phi(s)$, and apply the method of

" "Ueber die Methode des arithmetischen Mittels": Leipzig. Abh., Bd. xir., 1887.

† "La néthode de Neumann et le problème de Dirichlet": Acta Mlath., Bd. xx., 1896-97; "Sur les équations de la physique mathématique": Rend. del circolo Palermo, t. vir., 1894. 
Neumann, ${ }^{*}$ we are led to the expansion

$$
\phi(s)=\left[1+\lambda S_{\kappa}+\lambda^{2} S_{\kappa}^{2}+\ldots\right] f(s) .
$$

If $f(s)$ and $\kappa(s, t)$ are finite and integrable, the above series will have a finite radius of convergence different from zero, and will therefore give the solution for certain values of $\lambda$. Performing the operations, and changing the order of integration, the solution becomes

where

$$
\phi(s)=f(s)+\lambda \int_{a}^{b} K(s, t) f(t) d t,
$$

$K(s, t)=\kappa(s, t)+\lambda \int_{a}^{b} \kappa(s, r) \kappa(r, t) d r+\lambda^{2} \int_{a}^{b} \int_{a}^{b} \kappa(s, r) \kappa(r, \xi) \kappa(\xi, t) d r d \xi+\ldots$.

The function $K(s, t)$ is called the solving function of the integral equation. The similarity of the equations (7) and (8) shows that the relation betreen it and $\kappa(s, t)$ is a reciprocal one, and it also follows that

$$
\kappa(s, t)=K(s, t)-\lambda \int_{a}^{b} \kappa(s, r) K(r, t) d r ;
$$

so that $K(s, t)$ is the solution of (7) corresponding to $f(s)=\kappa(s, t)$.

It is noteworthy that the function $K(s, t)$ is also the solving function for the equation $t$

$$
\xi(t)=\psi(t)-\lambda \int_{a}^{b} \kappa(s, t) \psi(s) d s,
$$

the solution of this equation being given by

$$
\psi(t)=\xi(t)+\lambda \int_{a}^{b} K(s, t) \xi(s) d s .
$$

The disadvantage of the series obtained by Neumann's method is that it only converges for certain values of $\lambda$. This difficulty may be surmounted, as Plemelj shows, by the method of continuation; but a much better method was discovered by Fredholm $\ddagger$ in 1900, in which the function $K(s, t)$ is exhibited as the ratio of two power series which are convergent for all values of $\lambda$.

* Cf. Kellogg, " Zur Theorie der Integralgleichung $A(s, t)-\mathrm{A}(s, t)=\mu \int_{0}^{1} A(s, r) A(r, t) d r$ ": Göttinger Nachrichten, 1902, pp. 165-175.

† J. Plemelj, "Zur Theorie der Fredholmschen Funktionalgleichung": Wronatshefte für Mathematik und Physik, Bd. xv., 1904.

$\ddagger$ Ivar Fredholm, "Sur une nouvelle méthode pour la résolution du problème de Dirichlet": Oefversigt af kongl. vet. akad. Förh. Stockholm, 1900; also Acla Math., 1903. 
In this method the equation (7) is treated as the limit of a series of linear equations

$$
f_{1}=\phi_{1}-\lambda \sum_{1}^{n} \kappa_{1}, \phi_{r}, \quad f_{2}=\phi_{2}-\lambda \sum_{1}^{n} \kappa_{2 \cdot r} \phi_{r},
$$

and similarly for equation (10). The formula obtained for $K$ is then

$$
K(s, t)=-\frac{\Delta(\lambda ; s, t)}{\delta(\lambda)},
$$

where

$$
\begin{gathered}
\Delta(\lambda ; s, t)=-\kappa(s, t)+\lambda \Delta_{1}(s, t)-\lambda^{2} \Delta_{2}(s, t)+\lambda^{3} \Delta_{3}(s, t)-\ldots, \\
\delta(\lambda)=1-\delta_{1} \lambda+\delta_{2} \lambda^{2}-\ldots, \\
\Delta_{h}(s, t)=\frac{1}{h !} \int_{a}^{b} \ldots \int_{a}^{b} d s_{1} \ldots d s_{h}\left|\begin{array}{cccc}
\kappa(s, t) & \kappa\left(s, s_{1}\right) & \ldots & \kappa\left(s, s_{h}\right) \\
\kappa\left(s_{1}, t\right) & \ldots & \ldots & \ldots \\
\kappa\left(s_{h}, t\right) & \ldots & \ldots & \kappa\left(s_{h}, s_{h}\right)
\end{array}\right|, \\
\delta_{h}=\frac{1}{h} \int_{a}^{b} \Delta_{h-1}(s, s) d s, \quad \Delta_{0}(s, t)=\kappa(s, t) \\
\delta^{\prime}(\lambda)=\int_{a}^{b} \Delta(\lambda ; s, s) d s .
\end{gathered}
$$

If the last formula is combined with the previous one for $K(s, t)$, viz.,

$$
K(s, t)=\kappa(s, t)+\lambda \kappa_{1}(s, t)+\lambda^{2} \kappa_{2}(s, t)+\ldots,
$$

where $\quad \kappa_{1}(s, t)=\int_{u}^{b} \kappa(s, r) \kappa(r, t) d r, \quad \kappa_{n}(s, t)=\int_{1 !}^{b} \kappa(s, r) \kappa_{n-1}(r, t) d r$,

we get

$$
-\log \delta(\lambda)=a_{1} \lambda+\frac{1}{2} a_{2} \lambda^{2}+\frac{1}{3} a_{9} \lambda^{3}+\ldots
$$

where

$$
a_{1}=\int_{a}^{b} \kappa(s, s) d s, \quad a_{n}=\int_{a}^{b} \kappa_{n-1}(s, s) d s .
$$

Plemelj has used this result to obtain simpler expressions for $\delta(\lambda)$ and $\Delta(\lambda ; s, t)$,

$$
\begin{gathered}
\delta(\lambda)=1-\frac{\lambda}{1 !} a_{1}+\frac{\lambda^{2}}{2 !}\left|\begin{array}{ll}
a_{1} & 1 \\
a_{3} & a_{1}
\end{array}\right|-\frac{\lambda^{3}}{3 !}\left|\begin{array}{lll}
a_{1} & 1 & 0 \\
a_{2} & a_{1} & 2 \\
a_{3} & a_{2} & a_{1}
\end{array}\right|+\ldots, \\
\Delta(\lambda ; s, t)=-\kappa(s, t)+\frac{\lambda}{1 !}\left|\begin{array}{ll}
\kappa(s, t) & 1 \\
\kappa_{1}(s, t) & a_{1}
\end{array}\right|-\frac{\lambda^{2}}{2 !}\left|\begin{array}{lll}
\kappa(s, t) & 2 & 0 \\
\kappa_{1}(s, t) & a_{1} & 1 \\
\kappa_{2}(s, t) & a_{2} & a_{1}
\end{array}\right|+\ldots
\end{gathered}
$$

If the quantity $\delta(\lambda)$ which corresponds to the determinant of the system of linear equations does not vanish, there will be a unique solution of the integral equation, and this is given by the formulæ (8) and (13); 
but, if $\delta(\lambda)=0$, the homogeneous equation

$$
0=\phi(s)-\lambda \int_{a}^{b} \kappa(s, t) \phi(t) d t
$$

will possess a solution different from zero, and then certain conditions have to be satisfied by the function $f(s)$ in order that the equation (7) may possess a solution.* If $\lambda_{0}$ is a $p$-fold root of $\delta(\lambda)$, it can be shown that in the neighbourhood of $\lambda_{0}$ the function $K(s, t)$ has the form

$$
K(s, t)=\frac{P(s, t)}{\lambda-\lambda_{0}}+F(s, t),
$$

where $F(s, t)$ is finite for $\lambda=\lambda_{0}$ and $P(s, t)$ has the form

$$
P(s, t)=\Phi_{1}\left(s, \lambda_{0}\right) \Psi_{1}\left(t, \lambda_{0}\right)+\ldots+\Phi_{p}\left(s, \lambda_{0}\right) \Psi_{p}\left(t, \lambda_{0}\right) .
$$

The functions $\Phi_{1}, \ldots, \Phi_{p} ; \Psi_{1}, \ldots, \Psi_{p}$ are linearly independent solutions of the homogeneous equations

$$
\left.\begin{array}{l}
\phi(s)-\lambda_{0} \int_{a}^{b} \kappa(s, \theta) \phi(\theta) d \theta \\
\psi(t)-\lambda_{0} \int_{a}^{b} \kappa(s, t) \psi(s) d s
\end{array}\right\}
$$

respectively, and are connected by the orthogonal properties

$$
\int \Phi_{r}\left(t, \lambda_{0}\right) \Psi_{s}\left(t, \lambda_{0}\right) d t=\left\{\begin{array}{ll}
0 & (r \neq s) \\
1 & (r=s)
\end{array}\right) .
$$

It is useful to write the equation (7) in the symbolical form

$$
f(s)=S_{\kappa} \phi(s) \text {; }
$$

it can then be shown that the operations $S_{k}$ form a group, the law of multiplication being $S_{\kappa} S_{l}=S_{g}$, where

$$
g(s, t)=\lambda \kappa(s, t)+\lambda l(s, t)-\lambda^{2} \int_{a}^{b} \kappa(s, r) l(r, t) d r .
$$

When the function $\kappa(s, t)$ is symmetricalt in $s$ and $t$, many developments are suggested by the theory of the quadratic form $\Sigma_{\kappa_{p q}} x_{p} x_{q}$, which becomes in the limit the double integral

$$
\int_{a}^{b} \int_{a}^{b} \kappa(s, t) x(s) x(t) d s d t .
$$

The function $\delta(\lambda)$ then corresponds to the determinantal equation of the quadratic form, and Sylvester's theorem that the roots of such an equation

* See Fredholm, Acta Math., xxvu. ; also Erhard Schmidt, Inaugural Dissertation, Güttingen, 1905, p. 18.

† Hilbert, Göttinger Nachrichten, 1904.

OKR. 2. vor. 4. No. 918. 
are all real still remains true. The fact that a quadratic form can be expressed as a sum of squares also gives an important result, which may be stated as follows:-

Let $x(s)$ and $y(s)$ be two arbitrary functions such that $\int_{a}^{b}\{x(s)\}^{2} d s$ and $\int_{a}^{b}\{y(s)\}^{2} d s$ remain less than fixed quantities; then there exists an expansion

$$
\int_{a}^{b} \int_{a}^{b} K(s, t) x(s) x(t) d s d t=\sum_{n} \frac{1}{\lambda_{n}} \int_{a}^{b} \psi_{n}(s) x_{n}(s) d s \int_{a}^{b} \psi_{n}(s) y_{n}(s) d s .
$$

The function $\psi_{n}(s)$ is a solution of the homogeneous equation

$$
0=\psi_{n}(s)-\lambda_{n} \int_{a}^{b} \kappa(s, t) \psi_{n}(t) d t,
$$

and may be defined by the equation*

$$
\psi_{n}(s) \psi_{n}(t)=\frac{\Delta\left\{\lambda_{n} ; s, t\right\}}{\delta^{\prime}\left(\lambda_{n}\right)}
$$

These functions also possess the important orthogonal property

$$
\int_{a}^{b} \psi_{r}(s) \psi_{k}(s) d s=\left\{\begin{array}{ll}
0 & (r \neq s) \\
1 & (r=s)
\end{array}\right\} .
$$

It can be shown that every symmetrical function must possess at least one function $\psi, t$ and that a new solution of equation (7) may be obtained in terms of these functions in the form!

$$
\phi(s)=f(s)+\lambda \sum_{n} \frac{\psi_{n}(s)}{\lambda_{n}-\lambda} \int_{a}^{b} f(t) \psi_{n}(t) d t
$$

The definitions of the corresponding functions for an unsymmetrical Kern are given by Schmidt (l.c.): they are

$$
\left.\begin{array}{l}
\phi_{n}(s)=\lambda_{n} \int_{a}^{b} \kappa(s, t) \psi_{n}(t) d t \\
\psi_{n}(s)=\lambda_{n} \int_{a}^{b} \kappa(t, s) \phi_{n}(t) d t
\end{array}\right\} .
$$

- For an m-fold multiple root

$$
\left\{\frac{\frac{\partial^{m-1} \Delta(\lambda ; s, t)}{\partial \lambda^{m-1}}}{\frac{\partial^{m} \delta(\lambda)}{\partial \lambda^{m}}}\right\}_{\lambda=\lambda_{h}}=\psi_{h}(s) \psi_{h}(t)+\ldots+\psi_{h+m-1}(s) \psi_{h+m-1}(t) .
$$

The functions $\psi_{n}(s)$ are called by Hilbert the "Eigenfunktionen" of the "Kern" $\kappa(8, t)$, and the corresponding quantities $\lambda_{n}$ the " Eigenwerte."

+ If $\kappa$ is such that no solution of $\int_{a}^{b} \kappa(s, t) g(t)=0$ exists, there are an infinite number of functions $\psi$ (Hilbert).

‡ Erhard Schmidt, l.c. 
The quantities $\lambda_{n}$ are shown to be all real, and the following important expansion theorem is given :-

If $\kappa(s, t)$ is such that to every function $\alpha(s)$ and to every small quantity c there corresponds a function $\beta(s)$ such that

$$
\int_{a}^{b}\left[a(s)-\int_{a}^{b} \kappa(s, t) \beta(t) d t\right]^{2} d s<\epsilon,
$$

then every function $g(s)$ which can be defined by an integral of the form

$$
g(s)=\int_{a}^{b} \kappa(s, t) h(t) d t
$$

can be expanded in an absolutely and uniformly convergent series of the form $\Sigma A_{n} \phi_{n}(s)$, and a corresponding theorem holds for the functions $\psi$.

Another theorem for a quadratic form which has its analogue in the present theory is Gauss's variation problem, and Hilbert shows that the variation of the double integral

$$
\int_{a}^{b} \int_{a}^{b} \kappa(s, t) x(s) x(t) d s d t
$$

subject to the condition $\quad \int_{a}^{b}\{x(s)\}^{2}=1$,

leads to the integral equation

$$
0=x(s)-\int_{a}^{b} \kappa(s, t) x(t) d t .
$$

The theory of integral equations has an important application to the theory of linear differential equations of the second order.* If the Green's function corresponding to a given set of boundary conditions for the differential equation

$$
L(u)=\frac{d}{d x}\left(p \frac{d u}{d x}\right)+q(u)=0
$$

is taken as the generating function of an integral equation, the equation

$$
f(x)=\int_{a}^{b} G(x, \xi) \phi(\xi) d \xi,
$$

in which $f(x)$ is a function which can be differentiated twice, and which satisfies the given boundary conditions, will be given by

$$
\phi(x)=-L\{f(x)\} ;
$$

* Hilbert, “Grundzüge einer allgemeinen Theorie der linearen Integralgleichungen" (Zweite Mitteilung): Göttinger Nachrichten, 1904; Mason, Insugural Dissertstion, Göttingen, 1903 ; Andrae, Inaugural Dissertation, Güttingen, 1903. 
also the Green's function for the differential equation

$$
\Lambda(u)=L(u)+\lambda u=0
$$

is the solving function for the integral equation of the second kind

$$
f(x)=\phi(x)-\lambda \int_{a}^{b} G(x, \xi) \phi(\dot{\xi}) d \xi .
$$

The functions $\psi_{n}(x)$ corresponding to the function $G(x, \xi)$ are solutions of the equations $L(u)+\lambda_{n} u=0$, and so the well known expansions in terms of trigonometrical, Legendre, Bessel, and other functions are found to be particular cases of the general expansion theorem given above.

Another important application of integral equations is connected with Kiemann's problem in the theory of functions of a complex variable, but for this I must refer to the papers of Hilbert* and Kellogg. $\dagger$

The theory of integral equations depending on several variables and involving multiple integrals can be treated in a similar way and have similar applications. The formulæ are given in the dissertation of Andrae.

Fredholm has shown that the system of equations

$$
\phi_{\lambda}(x)+\int_{0}^{1} \sum_{\nu=1}^{n} f_{\lambda v}(x, y) \phi_{\nu}(y)=\psi_{\lambda}(x)
$$

can be reduced to one integral equation of the second kind by the following artifice :-

We define a function $F(x, y)$ for the values between 0 and $n$ by the $n^{2}$ conditions

and a function $\Psi$ by the $n$ conditions

$$
F(x, y)=f_{\lambda \nu}\{x-\lambda+1, y-\nu+1\} \quad \text { for } 0<\begin{aligned}
& x-\lambda+1 \\
& y-\nu+1
\end{aligned}<1,
$$

$$
\Psi(x)=\psi_{\lambda}(x-\lambda+1) \text { for } 0<x-\lambda+1<1 .
$$

If then the determinant of the equation

$$
\Phi(x)+\int_{0}^{n} F(x, y) \Phi(y) d y=\Psi(x)
$$

is different from zero, the solution is given by

$$
\phi_{\lambda}(x-\lambda+1)=\Phi(x) \text { for } 0<x-\lambda+1<1 .
$$

- " Über eine Anwendung der Integralgleichungen auf ein Problem der Funktionentheorie," Heidelberg Congress, 1904, p. 233 : Göttinger Nachrichten, Dritte Hitteilung, 1905.

+ "Unstetigkeiten bei der linearen Integralgleichangen mit Anwendung auf ein Problem ron Riemann": Math. Ann., Bd. Lx., p. 424. 
This remark is of some importance because an integral equation of the second kind in which the integral is taken along a complex path can be reduced to two equations of the above type by equating the real and imaginary parts, and so can be solved by means of Fredholm's formula.

Another equation which can be reduced to an integral equation of the second kind is the equation

$$
f(s)=\phi(s)-\lambda \int_{a}^{s} \kappa(s, t) \phi(t) d t,
$$

which has been solved by Volterra* by the method of Neumann. In order to reduce it to Fredholm's form, we define a function $f(s, t)$ which is equal to $\kappa(s, t)$ for $s>t$ and equal to zero for $b>t \geqslant s$. When Fredholm's formula is applied to the equation, it is found that the quantity $\delta(\lambda) \equiv 1$, and the solution reduces to that given by the method of Neumann: since $\delta(\lambda)$ is never zero, this solution will hold for all values of $\lambda$. The above equation is of importance in connection with the theory of linear differential equations, as will be shown in $\$ 3$ of this paper.

\section{Solution of the Integral Equation of the First Kind.}

The integral equation

$$
f(s)=\int_{a}^{b} \kappa(s, t) \phi(t) d t
$$

is not in general soluble for a perfectly arbitrary function $f$ : thus, if $\kappa(s, t)$ is a polynomial in $S$, the function $f(s)$ given by an equation of this kind can only be a polynomial.

In order, therefore, that the above equation may be soluble, we must restrict $f(s)$ to belong to a special class of functions associated with the generating function $\kappa$. There are two cases, however, in which the equation can be reduced to an integral equation of the second kind, and then $f(s)$ can have a high degree of arbitrariness.

1. Kellogg + has shown that the integral equation

$$
f(s)=\int_{0}^{1} \phi(t)[a \cot \pi(s-t)+S(s, t)] d t,
$$

where $S(s, t)$ is finite for $s=t$ and the integral is supposed to have

* Annali di Matemalica, 25, 1897.

† Math. Ann., Bd. corrr. 
its principal value, can be solved by means of Hilbert's formula

$$
\left.\begin{array}{l}
\phi(s)=\int_{0}^{1} f(t) \cot \pi(s-t) d t+\int_{0}^{1} \phi(t) d t \\
f(s)=-\int_{0}^{1} \phi(t) \cot \pi(s-t) d t+\int_{0}^{1} f(t) d t
\end{array}\right\} .
$$

For, if we multiply the above equation by $-\frac{1}{a} \cot \pi(r-s)$ and integrate from 0 to 1 , we get

$$
f_{1}(r)=\phi(r)+\int_{0}^{1} K(r, t) \phi(t) d t
$$

where

$$
\begin{aligned}
f_{1}(r) & =-\frac{1}{a} \int_{0}^{1} f(s) \cot \pi(r-s) d s, \\
K(r, t) & =-1-\frac{1}{a} \int_{0}^{1} S(s, t) \cot \pi(r-s) d s .
\end{aligned}
$$

Now this is an integral equation of the second kind, and so can be solved by means of Fredholm's formula.

2. When the function $\kappa(s, t)$ has a finite discontinuity at the point $s=t$. If we integrate the equation

$$
f^{\prime}(s)=\phi(s)+\int_{a}^{b} \kappa(s, t) \phi(t) d t
$$

between $a$ and $s$, it may be written

$$
f(s)-f(a)=\int_{a}^{b} R(s, t) \phi(t) d t,
$$

where the function $R(s, t)$ is such that

$$
\begin{aligned}
R(s, t) & =1+\int_{a}^{s} \kappa(s, t) d s & & \text { when } t<s \\
& =\int_{a}^{s} \kappa(s, t) d s & & \text { when } t>s .
\end{aligned}
$$

"Accordingly, the integral equation

$$
F(s)=\int_{a}^{b} R(s, t) \phi(t) d t,
$$

in which $F(a)=R(a, t)=0$, where $F(s)$ possesses a finite first derivate between $a$ and $b$, and $R(s, t)$ has a discontinuity +1 at the point $s=t$, but possesses a finite first derivate for all other values of $a$ and $b$, can be reduced to equation (4) and so solved by means of Fredholm's formula." 
3. We will now consider the equation

$$
f(s)=\int_{a}^{b} \kappa(s, t) \phi(t) d t,
$$

supposing that $\kappa(s, t)$ remains finite and integrable for all values of $t$ between $a$ and $b$, and for values of $s$ within a certain range.

We shall first examine the case in which $\kappa(s, t)$ is a symmetrical function of its arguments, and shall write the equation in the symbolical form

$$
f(s)=S_{\kappa} \phi(s) .
$$

The problem before us is to discorer the inverse operation

$$
\frac{1}{S_{\kappa}} f(s)=\phi(s) \text {. }
$$

Now we know that for an arbitrary $f(s)$ this can have no meaning, but for a function $f(s)$ which can be expanded in absolutely and uniformly convergent series of the functions $\psi_{n}(s)$ associated with $k(s, t)$ it has, and we may prove the following theorem :-

Theorem.-If $f(s)$ can be expanded in an absolutely and uniformly convergent series of the functions $\psi_{n}(s)$ that satisfy the homogeneous equations

$$
\psi_{n}(s)-\lambda_{n} \int_{a}^{b} \kappa(s, t) \psi_{n}(t) d t=0 \quad(n=1,2, \ldots),
$$

then it is possible to determine a function $\phi(t)$ such that

$$
f(s) \sim \int_{a}^{b} \kappa(s, t) \phi(t) d t
$$

may be less than any arbitrary small quantity $\epsilon$.

This may be regarded as the converse of the expansion theorem quoted in $\S 1$ : the proof is as follows :-

Let $f(s)$ and $\kappa(s, t)$ be finite and integrable for values of $s$ and $t$ lying between $a$ and $b$ : then the series

$$
F(t, x)=x S_{\kappa} f(t)-\frac{x^{3}}{1 !} S_{\kappa}^{3} f(t)+\frac{x^{5}}{2 !} S_{\kappa}^{5} f(t)-\ldots
$$

and

$$
H(s, x)=f(s)-\frac{x^{2}}{1 !} S_{\kappa}^{2} f(s)+\frac{x^{4}}{2 !} S_{\kappa}^{4} f(s)-\ldots
$$

are absolutely and uniformly convergent for all finite values of $x$. For, if $f$ is the maximum value of $|f(t)|$ and $k$ the maximum value of $|K(s, t)|$, the quantity

$$
\left|S_{\kappa}^{n} f(t)\right|<(b-a)^{n} f k^{n}
$$

and so each series becomes less than an exponential series. 
Now consider the function

$$
\phi(t)=2 \int_{0}^{M} F(t, x) d x
$$

where $M$ is very large. We have

$$
\int_{a}^{b} \kappa(s, t) \phi(t) d t=2 \int_{a}^{b} \kappa(s, t) d t \int_{0}^{M} F(t, x) d x=2 \int_{0}^{M} d x \int_{a}^{b} \kappa(s, t) F(t, x) d t .
$$

Now the series $F(t, x)$, being uniformly convergent, can be integrated term by term : accordingly we have

$$
2 \int_{a}^{b} \kappa(s, t) F(t, x) d t=\Sigma 2(-1)^{n} \frac{x^{2 n+1}}{(n) !} S_{\kappa}^{2 n+2} f(t)=-\frac{d}{d x} H(s, x) ;
$$

therefore

$$
\int_{a}^{b} K(s, t) \phi(t) d t=-\int_{0}^{M} \frac{d}{d x}[H(s, x)] d x=f(s)-H(s, M) .
$$

We must now find another expression for $H(s, M)$ which will indicate its value for large values of $M$. Since

$$
f(s)=\Sigma a_{n} \psi_{n}(s),
$$

and this series is uniformly convergent, we have

$$
\int_{a}^{b} \kappa(s, t) f(t) d t=\Sigma a_{n} \int_{a}^{b} \kappa(s, t) \psi_{n}(t) d t=\Sigma \frac{a_{n}}{\lambda_{n}} \psi_{n}(s),
$$

and this series is also uniformly convergent, since the $\lambda^{\prime}$ 's are all real and arranged in ascending order of magnitude. Operating again with $S_{\kappa}$, we have

similarly,

$$
S_{k}^{2} f(s)=\sum_{n=1}^{\infty} \frac{a_{n}}{\lambda_{n}^{2}} \psi_{n}(s)
$$

$$
S_{\kappa}^{r} f(s)=\sum_{n=1}^{\infty} \frac{a_{n}}{\lambda_{n}^{r}} \psi_{n}(s)
$$

Substituting in the series for $H(s, x)$, we have

$$
H(s, x)=\sum_{0}^{\infty}(-1)^{r} \frac{x^{2 r}}{r !} S_{\kappa}^{r} f(s)=\sum_{0}^{\infty}(-1)^{r} \frac{x^{2 r}}{r !} \sum_{1}^{\infty} \frac{a_{n}}{\lambda_{n}^{2 r}} \psi_{n}(s) .
$$

Now

$$
\sum_{m}^{\infty}\left|\frac{a_{n}}{\lambda_{n}^{r}} \psi_{n}(s)\right|<\frac{1}{\left|\lambda_{m}^{r}\right|} \sum_{m}^{\infty}\left|a_{n} \psi_{n}(s)\right|
$$

and, since the series $\sum_{0}^{\infty} a_{n} \psi_{n}(s)$ is absolutely convergent, we can find a number $m$ such that $\sum_{m}^{\infty}\left|a_{n} \psi_{n}(s)\right|<\epsilon$; therefore

$$
\sum_{m}^{\infty}\left|\frac{a_{n}}{\lambda_{n}^{r}} \psi_{n}(s)\right|<\frac{\epsilon}{\left|\lambda_{m}^{r}\right|},
$$


and so

$$
H(s, x)=\sum_{0}^{\infty}(-1)^{r} \frac{x^{2 r}}{r !} \sum_{1}^{m} \frac{a_{n}}{\lambda_{n}^{2 r}} \psi_{n}(s)+\eta,
$$

where

$$
|\eta|=\sum_{0}^{\infty} \frac{x^{2 r}}{r !}\left|\sum_{m}^{\infty} \frac{a_{n}}{\lambda_{n}^{2 r}} \psi_{n}(s)\right|<\sum_{0}^{\infty} \epsilon \frac{x^{2 r}}{\lambda_{m}^{2 r} r !}<\epsilon e^{x^{x /} / \mu_{m}^{2}}
$$

Rearranging the terms, we have

$$
H(s, x)=\sum_{1}^{m} a_{n} e^{-x^{2} / \lambda_{n}^{2}} \psi_{n}(s)+\eta ;
$$

and, making $\epsilon$ zero, we have finelly

$$
H(s, x)=\sum_{1}^{\infty} a_{n} e^{-x^{2} / \lambda_{n}^{2}} \psi_{n}(s) .
$$

Now, all the quantities $\lambda_{n}$ are real ; therefore it will be possible to find a number $p$ such that

$$
\left|\sum_{p}^{\infty} a_{n} e^{-x^{n} / \lambda_{n}^{2}} \psi_{n}(s)\right|<\left|\sum_{p}^{\infty} a_{n} \psi_{n}(s)\right|<\frac{\epsilon}{2} ;
$$

and, by choosing $M$ large enough, we can make

$$
\left|\sum_{1}^{p} a_{n} e^{-s^{2} / \lambda_{n}^{2}} \psi_{n}(s)\right|<\frac{\epsilon}{2} ;
$$

therefore $|H(s, M)|$ will be less than $\frac{1}{2} \epsilon+\frac{1}{2} \epsilon$, i.e., less than $\epsilon$. The integral $\int_{a}^{b} \kappa(s, t) \phi(t) d t$ will then differ from $f(s)$ by a quantity less than $\epsilon$, and so we should expect the solution of the integral equation to be given by

$$
\phi(t)=2 \int_{0}^{\infty} F(t, x) d x .
$$

Next consider the case in which $\kappa(s, t)$ is not necessarily symmetrical : we shall sssume that it is finite and integrable for values of $s$ lying betwoen $c$ and $d$, and for values of $t$ lying between $a$ and $b$.

Let the integral equation be

$$
f(s)=\int_{a}^{b} \kappa(s, t) \phi(t) d t,
$$

and consider the function

$$
g(s, r)=\int_{a}^{b} \kappa(s, t) \kappa(r, t) d t .
$$

It is evidently finite and integrable within the range $c$ to $d$, and is a symmetrical function of $r$ and $s$. Accordingly, the integral equation

$$
\psi(s)=\lambda \int_{c}^{d} g(s, r) \psi(r) d r
$$


will possess a number of solutions $\psi_{n}(s)$, and we may enunciate the following theorem :-

If $f(s)$ can be expanded in an absolutely and uniformly convergent series $\Sigma a_{n} \psi_{n}(s)$, then a function $\phi(t)$ can be determined so that

$$
\int_{a}^{b} \kappa(s, t) \phi(t) d t
$$

shall differ from $f(s)$ by as small a quantity as we please.

To prove this we construct the functions

$$
\begin{aligned}
& F(t, x)=x^{1} \int_{c}^{d} \kappa(s, t) d s \int_{a}^{b} \kappa(s, r) d r \int_{0}^{d} \kappa(\xi, r) f(\xi) d \xi \\
& -\frac{x^{3}}{1 !} \int_{c}^{d} \int_{a}^{b} \int_{c}^{d} \int_{a}^{b} \int_{c}^{d} \int_{a}^{b} \int_{c}^{d}(\ldots) \ldots+\frac{x^{5}}{2 !} \int_{c}^{d} \int_{a}^{b} \ldots-\ldots, \\
& H(s, x)=f(s)-\frac{x^{2}}{1 !} \int_{c}^{d} g(s, r) d r \int_{c}^{d} g(r, \xi) f(\xi) d \xi \\
& +\frac{x^{4}}{2 !} \int_{c}^{d} \int_{c}^{d} \int_{c}^{d} \int_{c}^{d}(\ldots)(\ldots) \ldots-\ldots, \\
& \phi(t)=2 \int_{0}^{M} F(t, x) d x .
\end{aligned}
$$

The proof then proceeds as before; for it is easy to see that these series sre absolutely and uniformly convergent for all finite values of $x$, and are connected by the relation

$$
2 \int_{a}^{b} \kappa(s, t) F(t, x) d t=-\frac{d}{d x} H(s, x) .
$$

The method just given is really of a much wider application; for it will sometimes apply to equations in which the integrals are taken along a complex path, or to integrals in which the limits are infinite, as, for instance, in the case of Fourier's theorem,

$$
\begin{aligned}
& f(s)=\int_{-\infty}^{\infty} e^{i s t} \phi(t) d t, \\
& \phi(t)=\frac{1}{2 \pi} \int_{-}^{\infty} e^{-i t x} f(x) d x .
\end{aligned}
$$

If either of the functions $f(s)$ or $\kappa(s, t)$ become infinite for a value of $s$ independent of $t$, we may make both sides finite by multiplying by a suitable function of $s$, and our method will still apply.

It should be noticed that, if the equation (8) can be solved for the 
generating function $\kappa(s, t)$, it can also be solved when the generating function is the solving function $K(s, t)$ of the equation

$$
\psi(s)=\chi(s)-\lambda \int_{a}^{b} \kappa(s, t) \chi(t) d t .
$$

For, since

$$
\chi(s)=\psi(s)+\lambda \int_{a}^{b} K(s, t) \psi(t) d t,
$$

we have

$$
\int_{a}^{b} \kappa(s, t) \chi(t) d t=\int_{a}^{b} K(s, t) \psi(t) d t .
$$

Accordingly, if a function $\chi(t)$ can be determined so that

$$
f(s)=\int_{.} \kappa(s, t) \chi(t) d t,
$$

the solution of

$$
f(s)=\int_{a}^{b} K(s, t) \psi(t) d t
$$

will, at the same time, be given by

$$
\psi(s)=\chi(s)-\lambda \int_{a}^{b} \kappa(s, t) \chi(t) d t .
$$

III. Reduction of the General Linear Differential Equation of the n-th Order to an Integral Equation of Volterra's Type.

Let it be assumed that the coefficients in the differential equation

$$
P_{x}(y) \equiv \frac{d^{n} y}{d x^{n}}+C_{1}(x) \frac{d^{n-1} y}{d x^{n-1}}+\ldots+C_{n}(x) y=0
$$

are such that for a given range of values of $x$ lying on a curve joining the points $a$ and $a+A$ in the complex $x$ plane the function $C_{s}(x)$ can be differentiated $n-s$ times with regard to $x$, and that all the quantities $C_{s}(x)$ and the derivatives assumed are finite and integrable for these values.

If, then, we multiply the equation by $x^{r}(r<n)$, and integrate along the curve from $\alpha$ to an intermediate point $x$, the result of the integration will be

$$
\left\{R_{x}\left[y(x) x^{r}\right]\right\}_{a}^{x}+\int_{a}^{x} y(x) p_{x}\left\{x^{r}\right\} d x=0,
$$

where $p_{x}(u)=0$ is the equation adjoint to $P_{x}(y)=0$, and $\dot{R}_{x}$ is the 
bilinear concomitant. This result follows at once from the relation*

$$
u P_{x}(y)-y p_{x}(u)=\frac{d}{d x}\left\{R_{x}(y, u)\right\} .
$$

Giving $r$ the values $0,1,2, \ldots, n-1$, we obtain $n$ equations from which the quantities $\frac{d^{n-1} y}{d x^{n-1}}, \ldots, \frac{d y}{d x}$ may be eliminated. The elimination may be performed by multiplying the equations by

$$
x^{n-1}, \quad-(n-1) x^{n-2}, \frac{(n-1)(n-2)}{2 !} x^{n-3}, \ldots,(-1)^{n-1}
$$

respectively and adding. For, if this is $\operatorname{don} \theta$, we get

$$
\left\{R_{t}\left[y(t),(x-t)^{n-1}\right]\right\}_{t=a}^{x}+\int_{a}^{x} y(t) p_{t}\left\{(x-t)^{n-1}\right\} d t=0,
$$

and, since $R_{t}(v, w)$ is of the form

$$
v \frac{d^{n-1} w}{d t^{n-1}}+d_{1} \frac{d^{n-2} w}{d t^{n-2}}+\ldots
$$

all the terms except those arising from $v \frac{d^{n-1} w}{d t^{n-1}}$ will vanish when $t=x$, and so the equation takes the form

$$
(n-1) ! y(x)-Q_{n-1}(x)+\int_{a}^{x} y(t) p_{t}\left\{(t-x)^{n-1}\right\} d t=0
$$

or

$$
Q_{n-1}(x)=y(x)+\frac{1}{(n-1) !} \int_{a}^{x} p_{t}\left\{(t-x)^{n-1}\right\} y(t) d t
$$

where $Q_{n-1}$ is a polynomial of degree $n-1$.

This is an equation of Volterra's type, and is exactly equivalent to the given differential equation; for the polynomial depends on the value of the $n-1$ derivates of $y$ at the point $\alpha$, and so contains $n$ arbitrary constants. An equation of a more general character bad previously been obtained by Dini, $\uparrow$ but it is not so simple as the one just given.

If the path of integration and all the coefficients are real, we may

- Forsyth's Theory of Differential Equations, Vol. Iv., p. 252.

† Ann. di Mat., Ser. 3, t. I., 1899, pp. 297-324; ib., t. II., 1899, pp. 125-183 : ib., t. Xr., Ser. 3, p. 385. 
apply the method of Neumann and obtain a converging series*

$$
\begin{aligned}
y(x)=Q_{n-1}(x) & -\int_{a}^{x} p_{t}\left\{\frac{(t-x)^{n-1}}{(n-1) !}\right\} Q_{n-1}(t) d t \\
& +\int_{a}^{x} d t p_{t}\left\{\frac{(t-x)^{n-1}}{(n-1) !}\right\} \int_{a}^{t} p_{s}\left\{\frac{(s-t)^{n-1}}{(n-1) !} Q_{n-1}(t) d t-\ldots\right.
\end{aligned}
$$

The series may also be written in the form

$$
y(x)=Q_{n-1}(x)-\int_{a}^{x} S(x, t) Q_{n-1}(t) d t,
$$

where

$$
\begin{aligned}
S(x, t) & =s_{0}(x, t)-s_{1}(x, t)+s_{2}(x, t)-\ldots \\
s_{i}(x, t) & =\int_{x}^{t} s_{i-1}(x, \xi) s_{0}(\xi, t) d \xi \\
s_{0}(x, t) & =\frac{1}{(n-1) !} p_{t}\left\{(t-x)^{n-1}\right\}
\end{aligned}
$$

The series for $S$ is certainly absolutely and uniformly convergent ; for it is what we should obtain by the application of Fredholm's formula, the determinant being in this case unity.

If, on the other hend, we want to pass from a point $a$ to any other point in the complex plane, we must choose a path of integration which does not pass through a singularity of one of the coefficients. We shall consider then the general equation

$$
f(z)=\phi(z)+\int_{a}^{z} \kappa(z, \xi) \phi(\xi) d \xi .
$$

Let $z$ and $\xi$ be expressed as functions of the arcs $s, \sigma$ of the curve joining $a$ to the two points $z$ and $\xi$ respectively, and write

$$
\begin{aligned}
\kappa(z, \xi) d \xi & =\left[F_{1}(s, \sigma)+i F_{2}(s, \sigma)\right] d \sigma, \\
\phi(z) & =\phi_{1}(s)+i \phi_{2}(s), \\
f(z) & =f_{1}(s)+i f_{2}(s) .
\end{aligned}
$$

We then have

$$
\left.\begin{array}{l}
f_{1}(s)=\phi_{1}(s)+\int_{0}^{s} F_{1}(s, \sigma) \phi_{1}(\sigma) d \sigma-\int_{0}^{s} F_{2}(s, \sigma) \phi_{2}(\sigma) d \sigma \\
f_{2}(s)=\phi_{2}(s)+\int_{0}^{s} F_{2}(s, \sigma) \phi_{1}(\sigma) d \sigma+\int_{0}^{s} F_{1}^{\prime}(s, \dot{\sigma}) \phi_{2}(\sigma) d \sigma
\end{array}\right\} .
$$

- The series is proved to be convergent for the general integral equation of this kind in Volterra's paper. 
We now adopt Fredholm's artifice and define a function $F(s, \sigma)$ for values of $s$ and $\sigma$ between 0 and $2 a$ as follows :-

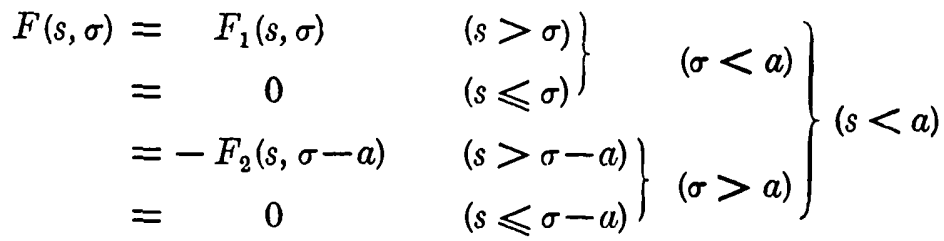

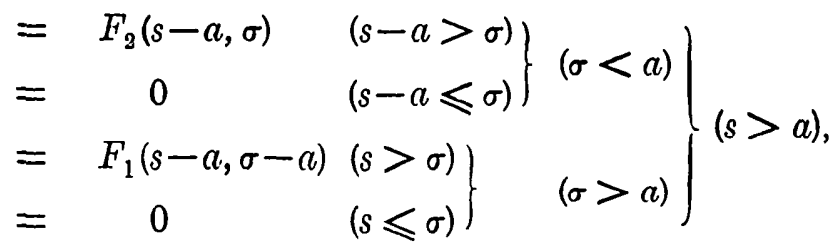

and two functions $g(s)$ and $h(s)$ such that $g(s), h(s)=f_{1}(s), \phi_{1}(s)$ or $f_{2}(s-a), \phi_{2}(s-a)$, according as $a$ is greater or less than $s$; the two equations may be replaced by the single equation

$$
g(s)=h(s)+\int_{0}^{2 a} F(s, \sigma) h(\sigma) d \sigma,
$$

and this may be solved by means of Fredholm's formula.

When we proceed to calculate $\delta(\lambda)$ by means of formula (21) it is found that all the quantities $a_{r}$ are zero; for in the integral

$$
a_{n}=\iint \ldots \int_{0}^{2 a} F\left(s, s_{1}\right) F\left(s_{1}, s_{2}\right) \ldots F\left(s_{n-1}, s\right) d s_{1} \ldots d s_{n-1}
$$

one at least of the factors $F$ will be such that its second argument is greater than its first, and will therefore be zero from the definition of $F$. The quantity $\delta(\lambda)$ reduces to unity, and so the method will certainly succeed. The solving function as given by formula (22) now reduces to the series obtained by applying the method of Neumann to equation (6), and, if we write down the series separately for $s$ greater and less than $a$. multiply the first by $\sqrt{ }(-1)$, and add, we are led to a result exactly analogous to formula (3). Accordingly we may apply the method of Neumann directly to equation (4), and the series obtained will certainly converge.

The theorem just proved is an existence theorem of a very general character. It states that a solution involving $n$ arbitrary constants exists at any point in the plane which can be reached by means of a curve at every point of which the quantity $p_{t}\left\{(x-t)^{n-1}\right\} d t$ is finite and integrable. Thus it is not necessary for all the coefficients in a linear differential equation to be continuous in order for a solution to exist. 
IV. The Effect of Varying the Limits in an Integral Equation of the Second Kind.

The quantities which occur in the integral equation

$$
f(s)=\phi(s)-\lambda \int_{a}^{b} \phi(t) \kappa(s, t) d t
$$

may be regarded as functions of $b$. Now, if $f(s)$ is independent of $b$, this equation may be written

$$
\phi(s, b)-\phi(s, a)=\lambda \int_{a}^{b} \kappa(s, t) \phi(t, b) d t ;
$$

hence the solution of the equation

$$
\psi(b)-\psi(a)=\int_{a}^{b} \chi(t) \phi(t, b) d t
$$

for $\psi(b)=\phi(s, b)$ is given by $\chi(t)=\lambda_{\kappa}(s, t)$.

The equation (2) is similar to equation (1) of $\S 1$, and so can be solved by the method of Volterra. We can therefore construct a function $\kappa(s, t)$, such that a given function $\phi(s, b)$ may be the solution of equation (1) for a certain range of values of $b$.

Again, if $\kappa(s, t)$ and $\phi(t, b)$ are supposed to be known, we may solve the equation (2) for any function $\psi(b)$ given by an equation of the form

$$
\psi(b)=\int_{s_{1}}^{s_{2}} \phi(s, b) \theta(s) d s .
$$

For, since the equation is linear, the solution will be

$$
\chi(t)=\lambda \int_{s_{1}}^{s_{2}} \kappa(s, t) \theta(s) d s .
$$

Next, consider the effect of varying $b$ in equation (1). If we differentiate the formula (17) of $\S 1$, viz.,

$$
\delta_{h}=\frac{1}{(h) !} \int_{a}^{b} \ldots \int_{a}^{b} d s_{1} \ldots d s_{h}\left|\begin{array}{cccc}
\kappa\left(s_{1}, s_{1}\right) & \kappa\left(s_{1}, s_{2}\right) & \ldots & \kappa\left(s_{1}, s_{n}\right) \\
\kappa\left(s_{2}, s_{1}\right) & & & \\
\vdots & & &
\end{array}\right|,
$$

we find that

$$
\begin{aligned}
& \frac{d \delta_{h}}{d b}=\frac{1}{(h-1) !} \int_{a}^{b} \ldots \int_{a}^{b} d s_{2} \ldots d s_{h}\left|\begin{array}{cccc}
\kappa(b, b) & \kappa\left(b, s_{2}\right) & \ldots & \kappa\left(b, s_{n}\right) \\
\kappa\left(s_{2}, b\right) & & & \\
\vdots & &
\end{array}\right| \\
& =\Delta_{h-1}(b, b) \text {. }
\end{aligned}
$$


Accordingly $\frac{d}{d b}[\delta(\lambda)]=-\lambda_{\kappa}(b, b)+\lambda^{2} \Delta_{1}(b, b)-\ldots$

$$
=\lambda \Delta(\lambda ; b, b)=-\lambda \delta(\lambda) K(b, b)
$$

or

$$
\frac{d}{d b}[\log \delta(\lambda)]=-\lambda K(b, b) .
$$

If we substitute in formula (19) of $\S 1$, and integrate with regard to $b$, we shall obtain

$$
\begin{aligned}
\log \delta(\lambda)= & -\lambda \int_{a}^{b} \kappa(s, s) d s-\lambda^{2} \int_{a}^{b} d s \int_{a}^{s} \kappa(s, r) \kappa(r, s) d r \\
& -\lambda^{3} \int_{a}^{b} d t \int_{a}^{t} \int_{a}^{t} \kappa(t, v) \kappa(r, s) \kappa(s, t) d r d s-\ldots,
\end{aligned}
$$

which may be identified with formula (20).

Again, if we differentiate the formula (19), since

$$
\kappa_{n}(s, t)=\int_{a}^{b} \kappa(s, r) \kappa_{n-1}(r, t) d r,
$$

we have

$$
\begin{aligned}
\frac{d}{d b} \kappa_{n}(s, t)=\kappa(s, b) \kappa_{n-1}(b, t) & +\int_{a}^{b} \kappa(s, r) \frac{d}{d b} \kappa_{n-1}(r, t) d r \\
= & \kappa(s, b) \kappa_{n-1}(b, t)+\int_{a}^{b} \kappa(s, r) \kappa(r, b) \kappa_{n-2}(b, t) d r \\
& \quad+\int_{a}^{b} \kappa(s, r) \int_{a}^{b} \kappa(r, \xi) \frac{d}{d b} \kappa_{n-2}(\xi, t) d \xi ;
\end{aligned}
$$

hence

$$
\frac{d}{d b} \kappa_{n}(s, t)=\kappa(s, b) \kappa_{n-1}(b, t)+\kappa_{1}(s, b) \kappa_{n-2}(b, t)+\ldots+\kappa_{n-1}(s, b) \kappa(b, t) .
$$

Therefore

$$
\begin{aligned}
\frac{d}{d b} K(s, t) & =\Sigma \lambda^{n}\left\{\Sigma \kappa_{r}(s, b) \kappa_{n-r+1}(b, t)\right\} \\
& =\lambda K(s, b) K(b, t) .
\end{aligned}
$$

We can now find the effect on a solution of equation (1). If $f(s)$ is independent of $b$, the formula

$$
\phi(s)=f(s)+\lambda \int_{a}^{b} K(s, t) f(t) d t
$$

will give $\frac{\partial}{\partial b} \phi(s)=\lambda K(s, b) f(b)+\lambda^{2} \int_{a}^{b} K(s, b) K(b, t) f(t) d t$

$$
=\lambda K(s, b) \phi(b) \text {. }
$$




\section{A certain Linear Partial Integral Equation.}

It was remarked in $\S 1$ that, if the function $\kappa(s, t)$ is the Green's function for the linear differential equation $L_{s}(u)=0$, the functions $\psi_{n}(s)$ connected with $\kappa(s, t)$ will be solutions of

$$
L_{s}(u)+\lambda_{n} u=0,
$$

and so the product $\psi_{n}(s) \psi_{n}(t)$, and the functions $\kappa(s, t), \kappa_{n}(s, t), K(s, t)$, which can in general all be expressed as a series of products of this kind, will be solutions of the partial differential equation

$$
L_{s}(u)=L_{t}(u) .
$$

In the general case when the function $\kappa(s, t)$ is no longer symmetrical, the functions $\kappa(s, t), \kappa_{n}(s, t)$ and $K(s, t)$ are all solutions of the partial integral equation

$$
\int_{a}^{b} \kappa(s, x) f(x, t) d x=\int_{a}^{b} \kappa(x, t) f(s, x) d x,
$$

which bears a striking analogy to (1).

To prove this we recall the fact that the relation between the functions $\kappa(s, t)$ and $K(s, t)$ is a reciprocal one; so that, since by formula (10) of $\S 1$ we have

$$
\kappa(s, t)=K(s, t)-\lambda \int_{a}^{b} \kappa(s, r) K(r, t) d r,
$$

we must also have

$$
K(s, t)=\kappa(s, t)+\lambda \int_{a}^{b} K(s, r) \kappa(r, t) d r ;
$$

for $\kappa(s, t)$ is the solving function for the equation (8). Combining these two equations, we get

$$
\int_{a}^{b} \kappa(s, r) K(r, t) d r=\int_{a}^{b} K(s, r) \kappa(r, t) d r ;
$$

so that $K(s, t)$ is a solution of (2). Equating the coefficients of $\lambda^{n}$ in this equation, we see that $\kappa_{n}(s, t)$ is also a solution of (2).

If we seek a solution of the form $\phi(x) \psi(t)=f(x, t)$, we are led to the equation

$$
\psi(t) \int_{a}^{b} \kappa(s, x) \phi(x) d x=\phi(s) \int_{a}^{b} \kappa(x, t) \psi(x) d x,
$$

which requires that $\phi(s)-\lambda \int_{a}^{b} \kappa(s, x) \phi(x) d x=0$,

$$
\psi(t)-\lambda \int_{a}^{b} \kappa(x, t) \psi(x) d x=0 .
$$

SER. 2. VOL. 4. NO. 919 . 
Now these equations can be satisfied for the same value* of $\lambda$, if $\lambda$ is a root of the equation $\delta(\lambda)=0$. Accordingly to each root of $\delta(\lambda)=0$ there corresponds a harmonic solution of the form $\phi(s) \psi(t)$.

If we can obtain a solution of (2) involving an arbitrary constant $\mu$, then the function obtained by multiplying by an arbitrary function of $\mu$ and integrating will also be a solution. The solving function $K(s, t, \lambda)$ of the integral equation

$$
f(s)=\phi(s)-\lambda \int_{a}^{b} \kappa(s, t) \phi(t) d t
$$

is such a solution. Accordingly (2) is satisfied by

$$
f(s, t)=\int_{\lambda_{1}}^{\lambda_{2}} K(s, t, \lambda) \chi(\lambda) d \lambda .
$$

If, however, another such function can be readily obtained, it may sometimes be used to obtain a convenient expression for $K(s, t)$.

\section{Literature.}

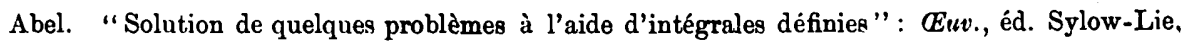
II., p. 11,1823 .

"Sur les fonctions génératrices et leurs déterminantes" : ilid., Ir., p. 67.

J. Liouville. " "Sur quelques Questions de Géométrie et de Mécanique, et sur un nouveau genre de Calcul pour résoudre ces Questions": J. de l'Ei. Pol., t. xIr., c. 21, 1832.

Riemann. "Ueber die Anzahl der Primzahlen unter einer gegebenen Grösse" : Werke, Weber, p. $140,1859$.

Rıuché. "Mémoire sur le calcul inverse des intégrales définies" : Par. C. R., 51, 1860, p. 126.

Laurent. "Calcul inverse des intégrales définies" : $J$. de math., $4,1878$.

Beltrami. "Intorno ad un teorema di Abel ad alcune sue applicazioni " : Lomb. Rend., 13, 1880. "La teorir delle funzioni potenziali simmetriche" : Bolognn Mcm. Accad., 2, 1881.

Sonine. "Sur la genéralisation d'une formule d'Abel": Acta Math., 4, 1884.

Pincherle. "Studi sopra alcune operazioni funzionali" : Bologna mem. accad., $7_{4}, 1886$.

__ "Sur certaines opérations fonctionuelles": Acta Math., 10, 1887.

"Sur la génération de systèmes récurrents au moyen d'une équation linéaire différentielle": Acta Math., 16, 1893.

___ “Sulle equazioni funzionali lineari" : Rom. Linc. Rent., 14, Oct., 1905.

Neumann. "Ueber die Methode des urithmetischen Mittels"' : Leipz. Abh., Bd. xm., 1887.

Du Bois Reymond. "Bemerkungen über $\Delta z=0$ " : J. für Mat., Bd. crr., 1888.

Volterra. "Sopra un problema di elettrostatica": Rom. Linc. Trans., 8, 8, 1895.

"Sulle inversioni degli integrali definiti" : Rom. Linc. Rend., $5_{5}, 1896$; Tor. A., $5_{5}, 1896$

(4 pupers).

"Sopra alcune questioni di inversioni di integrali definiti" : Ann. di Mat., 2, 25, 1897.

Levi Civita. "Sull' inversione degli integrali definiti": Tor. A., 31, 1895.

"I gruppi di operazioni funzionali e l'inversione degli integrali definiti " : Lomb. Rend., $28,1895$.

Le Roux. " "Sur les intégrales des équations linéaires," p. 243 : Ann. Ec. Norm., 2, 12, 1895. "Sur une inversion d'intégrale double": Par. C. R., 130, 882-884, 1900. 
Fredholm. "Sur une nouvelle méthode pour la résolution du problème de Dirichlet ": Oefversigt af kongl. vet. akad. Förh. Stockholm, 1900.

"Sur une classe de transformations rationnelles": Par. C. R., 1902, p. 219.

"Sur une clasee d'équations fonctionnelles": ibid., p. 1561; Acta Math., 27, 1903.

"Sur la théorie des spectres" : Par. C. R., 1906, p. 505.

Holmgren. " Sur un théorème de M. Volterra sur l'Inversion des Intégrales définies," Tor. A., $35,1900$.

“Recherches sur l'inversion des intégrales définies": $D_{p s .} A$., III., 1900.

Kellogg. "Zur Theorie der Integralgleichungen und des Dirichlet'schen Princips": Diss. Gött., 1902.

"Zur Theorie der Integralgleichung": Gött. Nachr., 1902, pp. 165-175.

" Unstetigkeiten in den linearen Integralgleichungen": Math. Ann., Bd. LVIrr.

" Unstetigkeiten bei den linearen Integralgleichungen mit Anwendung auf ein Problem von Riemann" : Math. Ann., Bd. xx., p. 424.

Muson. "Randwertaufgaben bei gewöhnlichen Differentialgleichungen": Diss. Gött., 1903.

"Sur les solutions satisfaisani à des conditions aux limites dounées de l'équation différentielle" : J. de Math., 10

“Green's Theorem and Green's Functions for certain Systems of Differential Equations" : Ainer. Trans., Vol. v., No. 2, p. 220, 1904.

Andrae. “Hilfsmittel zu einer allgemeinen Theorie der linearen elliptischen Differential. gleichung 2. Ordnung" ": Diss. Gött., 1903.

Hilbert. "Grundzüge einer allgemeinen Theorie der linearen Integralgleichungen": Gött. Nachr., 1904, Hefte 1 und 3; 1905, Heft 4.

“Über eine Anwendung der Integralgleichungen auf ein Problem der Funktionentheorie" : Heidl. Congr., 1904, p. 233.

Plemelj. "Über die Anwendung der Fredholm'schen Funktionalgleichung in der Potentialtheorie": Wien. Ber., 112, March, 1903.

"Zur Theorie der Fredholmschen Funktionalgleichung": Monatsh. für Math., xv., 1904.

Schmidt. "Entwicklung willkürlicher Funktionen nach Systemen vorgeschriebener": Diss. Gött., 1905.

Cailler. "Théorème d'inversion d'intégrale définie constituant une extension naturelle des intégrales de Fourier" : Arch. sc. phys., (4), II., 97, 98, 1901.

Cesàro. "Sopra un' equazione funzionale trattata da Beltrami": Nap. Rend. (3), 7, p. 284, 1901.

Burgatti. "Sull'inversione degli integrali definiti": Rom. Lin. Rend. (5), 12. pp. 443 and 596.

Goursat. "Sur un problème d'inversion résolu par Abel ": Acta Math., 27, 1903.

Lerch. "On a Point in the Theory of Abel's Generating Functions": Acta Math., 27, 1903.

Dini. “Studii sulle equazioni differenziali lineari" : Ann. di Mat., IIr., t. xr., p. 385, 1905.

Zaremba. "Contribution à la Théorie d'une Equation fonctionnelle de la Physique" : Pal. Rend., t. xux., 1905.

E. Picard. "Sur une équation fonctionnelle": Par. C. R., 1904, p. 245. 